http://dx.doi.org/10.12795/RAA.2012.i03.07

\title{
JUVENTUDES Y MIGRACIONES EN ITALIA. DECONSTRUYENDO LA POSTERIDAD INOPORTUNA
}

\author{
LUCA QUEIROLO PALMAS \\ Università di Genova - Universidad de Lleida (Marie Curie Fellowship)
}

\section{Resumen.}

El texto reconstruye el debate académico mas reciente en Italia sobre el tema de la juventud migrante; los hijos de las migraciones, llevando el estigma de su posteridad inoportuna, son a menudo pensados como un desafío a la cohesión social, sujetos definidos en primera instancia por su déficit y su pasividad, marcados por el comunitarismo étnico, y considerados problemáticos porque se encuentran suspendidos entre universos culturales. El enfoque aquí propuesto quiere, por el contrario, explorar la importancia de una lectura construccionista del objeto capaz al mismo tiempo de rescatar las dimensiones de agencia y resistencia de los sujetos.

Palabras clave: Jóvenes, Italia, migraciones, agencia, racismo, segundas generaciones

\section{Abstract.}

The article explores the more recent academicals debates about young people with migrant origin in Italy. In the mainstream theoretical approaches the child of migration, carrying the stigma of a inopportune desire to settle, are often thought as a challenge to the social 
order, as a passive subjects marked by a cultural and behavioural deficit, as a victims of a ethnic communitarism, as a problematic presence because of their suspension among different cultures. Our perspective, at the opposite, pretends to show the significance of a constructionist approach in order to put emphasis on the dimension of youth agency and resistance in a context of a post-migratory society.

Keywords: Youth, Italy, migrations, agency, racism, second generations

\section{1. ¿EL DÉFICIT DE QUIEN ESTÁ DIVIDIDO ENTRE CULTURAS?}

En el número monográfico reciente de "Libertá civili" [libertades civiles], la revista dedicada al tema de la segunda generación publicada por el Ministerio del Interior italiano, se motiva la propuesta de introducir un mecanismo de doble ciudadanía por el siguiente argumento: "[...] también para tener en cuenta su singularidad que es la de pasar en un tiempo muy corto de hijos de emigrantes, pero ciudadanos del país de origen, a hijos de inmigrantes, pero extranjeros en el país de llegada; y entonces tener que encargarlos, en muchos casos, a un servicio de psicoterapia transcultural especifico para chicos migrantes (del cual hay ya algún ejemplo en Italia). Eso para asegurarles un futuro menos problemático y, a continuación, capacitarlos para ser padres atentos y concienciados" (Golini, 2011: 19).

La contribución de Antonio Golini, uno de los más estimados demógrafos italianos, más allá de su propuesta de ampliar las mallas de la ciudadanía, que sin duda se puede compartir, me parece interesante por su capacidad de revelar la doxa (Bourdieu, 2003) a través de la cual el pensamiento docto, de Estado y de sectores mayoritarios de la opinión pública, define la irrupción de una cuestión juvenil en el campo de las migraciones.

Los menores de origen extranjero están así pensados como una comunidad articulada en la dimensión étnico-cultural, su presencia cada vez mayor representa un reto para la cohesión social para las sociedades receptoras, mientras que la relativa condición de vulnerabilidad depende del estar divididos entre dos culturas; de aquí sigue la propuesta de que unos servicios de psicoterapia transcultural se encarguen de ellos. El conjunto de estas dimensiones configuraría según un sociólogo prominente, "una bomba de relojería" (Barbagli, 2002).

Aquí vemos en acción, pues, un conjunto de estructuras profundas de percepción y clasificación social sobre estos segmentos de la población: sujetos con un déficit y una discapacidad cultural (que se refleja principalmente en el rendimiento académico), la culturización / etnización justificada sobre la base de estas insuficiencias, la negación de una dimensión activa, de agencia, como propiedad y oportunidad en la vida de los hijos de las migraciones, y una pretensión de asimilación lineal como garantía de orden social (se abandona de hecho una cultura-nación para ser acogidos en otra cultura-nación). 
Estas estructuras profundas corresponden al re-nacimiento de la que Ambrosini (2009) ha definido "la ansiedad de asimilación de las sociedades receptoras". Estructuras de pensamiento que atraviesan, además de los debates políticos y los dispositivos de gestión administrativa de las migraciones, el campo mismo de los migration studies, donde se comparan permanentemente con otras miradas, otras líneas de investigación, otras adquisiciones de saber empírico. En esta aportación nos proponemos revisar críticamente algunas construcciones - principios de visión y división - siguiendo a Bourdieu - en relación con las segundas generaciones y, más en general, a los hijos de la inmigración, debatiendo con las contribuciones teóricas y las investigaciones que en los últimos años han desafiado a la ciencia normal de las migraciones.

\section{LA POSTERIDAD INOPORTUNA}

Hoy en día la presencia de los hijos de la inmigración, así como de la inmigración en general, es un hecho estructural para la sociedad italiana, impulsado tanto por las dinámicas de las reunificaciones como por el efecto de los nacimientos. Los menores extranjeros en Italia en el año 2010 alcanzaban el millón. Aún más interesante es observar que el $58 \%$ de los niños legalmente residentes habían nacido en Italia, mientras que otro 21\% había llegado antes de los 5 años de edad (Barban et al., 2011); empleando el lenguaje de tipo decimal acuñado por Rumbaut $(1997)^{1}$, al día de hoy en Italia, el 80\% de los niños extranjeros se refiere a la generación 2.0 y 1.75 . Es decir, en 8 casos de 10 llamamos inmigrantes a niños, adolescentes y jóvenes que no han realizado ningún tipo de migración o para los que el viaje y la partida representan una experiencia de la primera infancia sustentada por los recuerdos familiares, además de por la mirada excluyente de la sociedad receptora. Una variedad de pruebas demuestra la solidez del proceso de estabilización y enraizamiento social de los flujos (la natalidad y las reagrupaciones, las inscripciones escolares, la difusión de las parejas mixtas, la inversión inmobiliaria y la bancarización de las familias migrantes), que nos invitan a considerar Italia como una sociedad post-migratoria (Martiniello, 2000).

1. Según Rumbaut, los nacidos en el país receptor de padres extranjeros representan la generación 2.0, la generación 1.5 sería aquella para la cual la socialización escolar y familiar se ha llevado a cabo más o menos de igual manera entre el país de origen y país de llegada, mientras que la generación 1.75 corresponde a aquel segmento de hijos de la migración llegados en la primera infancia y que han llevado a cabo la mayor parte del currículo escolar en el país de llegada. Por último en la generación 1.25 se encuentran los jóvenes que han emigrado entre los 13 y 17 años. Al igual que cualquier tipología, su límite es la rigidez; su valor es considerar el efecto combinado de nacimiento, antigüedad de residencia y frecuencia escolar. Se trata obviamente de agregados estadísticos y no de grupos de por sí. 


\section{Cuadro 1 - Algunos indicadores de posteridad de las migraciones}

\begin{tabular}{|l|c|}
\hline Menores Extranjeros (2010) & 933.693 \\
\hline Incidencia sobre el total de la población extranjera residente & $22 \%$ \\
\hline Menores extranjeros nacidos en Italia (2010) & $58 \%$ \\
\hline Nuevos nacimientos en Italia de ambos padres extranjeros (2009) & 77.109 \\
\hline Incidencia sobre el total de los nuevos nacimientos (2009) & $13,6 \%$ \\
\hline Alumnos extranjeros (2009/2010) & 673.592 \\
\hline de los cuales nacidos en Italia & $37 \%$ \\
\hline $\begin{array}{l}\text { Incidencia de los alumnos extranjeros sobre el total de los alumnos } \\
(2009 / 2010)\end{array}$ & $7.5 \%$ \\
\hline Alumnos extranjeros en las escuelas de infancia (2008/2009) & 125.059 \\
\hline $\begin{array}{l}\text { de los cuales nacidos en Italia } \\
\text { (2010) }\end{array}$ & $73,3 \%$ \\
\hline \begin{tabular}{l} 
Estudiantes extranjeros matriculados en la universidad (2009/2010) \\
\hline \begin{tabular}{l} 
Concesiones de ciudadanía (2010) \\
\hline
\end{tabular}
\end{tabular} & 58.213 \\
\hline
\end{tabular}

Fuente: Caritas Migrantes, 2010, Istat 2011, Ministero dell'Interno 2011. Elaboración: propia

Estos procesos de enraizamiento material están atrapados en una retorica de la emergencia (alarmas por la llegada incontrolada de refugiados, coyotes, pateras, clandestinos, aulasguetos, etc...) y en una consiguiente gestión administrativa de las migraciones en términos de reproducción de la precariedad y de la negación tanto del derecho a quedarse como del derecho a moverse. Como sugestivamente señala Saitta (2011), la condición migrante en Italia, y por extensión también la de los hijos de los migrantes, sigue oscilando todavía después de 30 años del comienzo de los primeros flujos importantes entre Kafka y el Carnaval: un castillo de burocracia kafkiana vejatoria y de operaciones policiales eficaces más simbólicamente que materialmente (desde la ciudadanía de sangre a la dificultad para naturalizarse, desde la lucha contra los matrimonios irregulares a los obstáculos para la reagrupación, desde el arranque de los bancos en los espacios públicos a la introducción del delito de inmigración ilegal, desde los alcaldes sheriffes a las patrullas cívicas, desde las disposiciones municipales en contra del kebab a las dificultades para las inscripciones en los registros de nacimientos), amplificado de forma grotesca en un 
carnaval de una nueva producción imaginada de la nación: la producción de un ellos, con rasgos insidiosos y perturbadores, para reafirmar la existencia de un nosotros. En las palabras de Saitta "[...] el estado actual del discurso sobre los migrantes corresponde en gran parte a una dimensión constitutiva: pone en escena el momento exacto en que se lleva a cabo la producción de una comunidad. En otras palabras, el esfuerzo permanente de las élites italianas, como de las elites de muchos otros países, de volver a proponer el momento fundacional de la comunidad nacional. Para ser francos, lo que hace el caso italiano complejo y fascinante, es este carnaval, o sea, el simulacro de una fundación nacional que nunca ha verdaderamente tenido lugar y que, por lo tanto, debe ser periódicamente reproducida" (Saitta, 2011:2).

En un documental reciente sobre las culturas juveniles en la Europa mestiza (Diaco et al., 2009) se puede advertir como actúa esta dimensión kafkiana que viven sobre su piel los hijos de la inmigración: un grupo de amigos y coetáneos crecidos juntos en un barrio de la periferia, cuyo estatus jurídico se desarrolla de forma esquizofrénica entre la ciudadanía, la irregularidad, los riesgos de expulsión, la serie de autorizaciones de residencia que hay que renovar. El carnaval se despliega en los desfiles multiculturales (Baumann, 2003) a través de los cuales se celebra una etnicidad falsa, paradójicamente funcional a la revitalización de la idea de un nosotros, diferente y superior culturalmente.

Por estas razones, en la mirada de los nativos, los hijos de los migrantes, que se han vuelto presencias estables en las ciudades europeas, encarnan lo que Sayad (2002, 2008) definía como la posteridad inoportuna, dado que rompen el mito de la temporalidad de la migración y cuestionan el rol del migrante como invitado bueno, neutral y silencioso. Representan una proyección hacia el futuro, haciendo permanente lo que se pensaba provisorio, derribando de esta manera el sueño y el mito del retorno que aúna el pensamiento del Estado y las expectativas de los migrantes de primera generación. Los hijos de inmigrante son entonces, para el pensamiento y el cuerpo de la nación, el emblema de una inmigración sufrida, el fantasma de todas las retóricas contemporáneas sobre el derecho de los Estados a elegir qué inmigración aceptar.

\section{3. ¿CUÁNDO SE DEJA DE SER INMIGRANTES? LAS ETIQUETAS COMO CAMPO DE LUCHAS SIMBÓLICAS}

No está claro, sin embargo, quién tiene que ser o no incluido en la categoría de segunda generación. ¿Qué es lo que importa? ¿El lugar de nacimiento, el hecho de descender de padres extranjeros, las características de la socialización, la experiencia escolar, una mezcla de todos estos factores? Como hemos señalado, Rumbaut subraya la importancia del lugar de nacimiento y el tiempo de socialización en la sociedad receptora en su propuesta de definición decimal de segundas generaciones, mientras que Ambrosini (2005), de forma pragmática, abre los límites de la categoría a todos los hijos de la migración. 
El nudo de la cuestión, sin embargo, se encuentra más en profundidad: por un lado el hecho de insistir en la origen migrante de esta generación pone de relieve una cualidad que parece surgir de una definición casi ontológica del ser de los sujetos incluidos y marcado por esta etiqueta (efecto de reificación), lo que hace estas vidas incomparables con las de los coetáneos nativos (efecto de remoción de una condición similar de la juventud), reduciendo una biografía a un origen (efecto metonímico) y transformando, en fin, el origen en el destino (efecto de sobredeterminación).

La categoría segunda generación, haciendo hincapié en la conservación de la distancia cultural, recuerda a los jóvenes de familias inmigrantes y de clases populares que, a pesar de sus esfuerzos, seguirán estando siempre vinculados a otro espacio cultural, y por lo tanto su existencia no es digna de convertirse en una ciudadanía plena. Al mismo tiempo, hay que reconocer que la omnipresencia del término segundas generaciones en los debates contemporáneos y en las mismas aportaciones subjetivas hechas por los hijos de la migración ha contribuido a destacar el nacimiento de una nueva cuestión juvenil: "la existencia y el oxímoron", tomando a préstamo las retoricas de unas de las principales asociaciones de las segundas generaciones (Red G2), de quien es italiano con una autorización de residencia.

Como subraya Delgado (2010), toda terminología acerca de la migración - inmigrantes, migrantes, segundas generaciones - se explica por los efectos en términos de poder que quiere producir: definir marcos étnico-culturales que pueden naturalizar, ofuscar, legitimar la desigualdad estructural. ¿En qué lógica se puede aplicar a ciertas categorías de personas un participio pasado objetivado (inmigrado)? "El extranjero en este caso se entiende y se reconoce como alguien que ha llegado pero queda petrificado en el tiempo, atrapado en su pasado, esclavo del momento especifico en que ha alcanzado su hipotética destinación, un momento que no podrá sobrepasar en ninguna circunstancia" (Delgado, 2010: 27).

Y en paralelo ¿cómo puede ser posible hablar de migrantes, o sea "utilizar un participio presente para una acción en el pasado" (ibid., 2010)? ¿Sobre la base de qué lógica es posible hablar de menores inmigrantes en Italia, cuando en 8 casos de 10 estos sujetos nacieron, o se criaron desde la infancia en Italia? ¿En virtud de las disposiciones de ciudadanía, no deberíamos tal vez, más apropiadamente, hablar de extranjeros no inmigrantes, una pareja de términos que inmediatamente suena contradictoria? ¿Y por último, la cualidad de migrante, se hereda, se transmite de padre a hijo, como parecería aludir el término segundas generaciones?

Parece importante, entonces, desplazar el plano de la observación hacia la pluralidad de procesos que se articulan en torno a la definición y a la imagen de un grupo social, verdaderas luchas simbólicas entre hetero-representaciones y auto-representaciones. Por un lado está el intento de establecer una esencia, culturalizandola, etnizándola, 
inferiorizándola: no hay que interrogarse sobre las segundas generaciones, sino cómo determinados sujetos están construidos como segundas generaciones y sobre los efectos del régimen del discurso en términos de estrategias y prácticas que los sujetos marcados ponen en marcha en la presentación pública del yo. Retomando a Delgado: "Para ganarse la marca de migrante o inmigrante es imprescindible también ser pobres, intrusos, peligrosos, excesivos, inferiores, atrasados, discapacitados [...] Así que el inmigrante no es solo una pieza fundamental de un sistema de producción fundado en la explotación y la necesidad de un recambio-renovación de la población; él asume sobretodo el carácter de un operador simbólico, haciendo materialmente representable [...]la desorganización social vista desde el interior" (Delgado, 2010: 27).

En el caso de los hijos de la migración, esta operación simbólica y material se enfrenta con la dimensión de la posteridad, con el derrumbe del mito del retorno, con la que ha sido definida como la paradoja de la integración (Wrench, Rea y Ouane, 1999): la percepción de un surplus ilegítimo de discriminación, porque se ha estado expuesto a los dispositivos de la socialización que operan en el seno de la sociedad receptora, el choque entre expectativas y oportunidades, la internalización de los objetivos sociales (hablando en los términos de Merton) y la dificultad de encontrar medios adecuados para alcanzar esos mismos objetivos compartidos .

Este conjunto de elementos precipita en un rechazo cada vez mayor de los mecanismos de la integración subalterna (Ambrosini, 2005) o de la inclusión diferencial (Mezzadra, 2004). Esta negativa puede basarse en diferentes estrategias empleadas por los sujetos enmarcados en el estigma de la migración; la petición de superar definitivamente la posibilidad de ser italianos con autorización de residencia, la reivindicación de nuevas hyphenated identities - ¿cuando podrá existir, se pregunta Andall (2003 a), un negroitaliano?- pero también la ironía, el camuflaje, la gestión táctica de la diferencia en el marco del multiculturalismo cotidiano (Colombo y Semi, 2007).

Volviendo a la pregunta con la que se abre este subcapítulo, estas categorías de personas definidas desde arriba, dejan de ser inmigrantes / migrantes / de origen inmigrante, sólo cuando consiguen a acceder a aquella prerrogativa de la ciudadanía que en palabra de Goffman podemos llamar como desatención cortés, o en palabras de Delgado (2007: 192) derecho a la indiferencia, o sea una condición de opacidad por la cual algunos sujetos no "son forzados a explicar, justificar lo qué hacen, piensan, cuáles son los ritos en los que participan, qué comen, qué sexualidad tienen, qué sentimientos religiosos profesan, cuál es la visión del mundo en que creen, todos los datos que nosotros, los normales, nos negaríamos a proporcionar a las personas extrañas al núcleo más íntimo de nuestras relaciones ". Reivindicar para todos y todas el derecho a la opacidad se vuelve entonces la condición de generar formas de encuentros menos asimétricas y no subalternas a una mirada colonial (Glissant, 2007). 


\section{4. "ES DIFÍCIL SER DE MI GENERACIÓN EN ITALIA. NUESTROS PADRES TUVIERON QUE LUCHAR POR UN TRABAJO, NOSOTROS DEBEMOS LUCHAR PARA VIVIR"}

Con estas palabras, Aline, una joven italo-congoleña que se crió en Roma, entrevistada en el marco de una investigación sobre las segundas generaciones en Europa, nos expone la diferencia entre padres e hijos en los procesos migratorios: la vida como algo que va más allá de la lógica de la utilidad y de la obtención de un puesto de trabajo, dispositivos constituyentes de la figura del inmigrante de primera generación.

Luchar para vivir significa tener la oportunidad de estudiar, viajar, elegir la forma de representar el self en los diferentes contextos de la vida cotidiana. Luchar para vivir significa salir del estatus jurídico-administrativo de la autorización de residencia - ¿se puede estar autorizado a vivir en el lugar donde se ha nacido y se ha crecido? - Pero también significa escaparse del discurso sobre la integración, el criterio desde el cual los migrantes y los hijos de las migraciones son recurrentemente llamados a compararse.

Esta palabra - integración - a menudo se convierte en el espejo y la coartada de la exclusión, como nos ha recordado el grito y la invocación de Ahmed Djouder, en un texto que tuvo amplia difusión en Francia después de los disturbios en los suburbios: "Y luego escuchaos un poco mientras utilizáis esta palabra, integración. Esta palabra es débil. ¿Cómo se puede ser tan torpe? Fijaos en cómo revela vuestra mala fe. Pedir que nos integremos después de haber estado aquí desde dos o incluso cuatro generaciones es una verdadera tomadura de pelo. ¿Vosotros creéis que integrándonos vais a conseguir domar los suburbios, reducir la delincuencia? Dicho entre nosotros, los franceses aman esta palabra, integración, porque les hace creer que son capaces de domesticarnos. Pero, no somos animales salvajes. ¿Lo sabíais?... Vosotros habéis invertido los papeles. No nos corresponde a nosotros hacer el esfuerzo. Hace demasiado tiempo que nos rompemos el culo arreglando vuestras viejas calles con el martillo neumático, montando los ferrocarriles de vuestros trenes con el soplete o poniendo los azulejos nuevos sobre el hormigón de vuestro cuarto de baño. No nos vamos a integrar, porque esta palabra es repugnante. Sabe a campo de concentración... Nosotros no esperamos con falsa avidez que vosotros nos aceptéis. Vuestra integración nos hace reir. Es una palabra tremenda. No nos importa. Nosotros no tenemos que integrarnos. Nosotros no vamos a integrarnos. Esperaremos que vosotros reaccionéis, que nos miréis como otro cualquiera, como un extranjero cualquiera como un francés cualquiera" (Djouder, 2007: 91).

En una reciente propuesta del gobierno italiano, el acceso a la autorización de residencia se pauta y se ajusta a través de un acuerdo de integración. La vida por puntos: puntos que se pierden por condenas penales y otros delitos, puntos que se ganan a través de cursos de educación a la ciudadanía, títulos educativos y conocimientos de la lengua italiana. La legitimidad de la presencia debe ser entonces merecida, demostrada. 
Aline, en virtud de sus estudios universitarios y su perfecto conocimiento del italiano podría, sin duda, ganar el derecho a una autorización de residencia a largo plazo, librándose del ritual degradante de las colas en los mostradores de las comisarías de policía, mientras que los jóvenes del subproletariado migrante, expulsados por los circuitos formativos, enredados en las mallas de redes étnicas de baja movilidad social, en permanentemente oscilación entre la economía informal y la economía formal, aunque hayan crecido en Italia, estarían destinados a enfrentarse con la espada de Damocles de la deportación hacia los países de origen de las familias, lugares con los que mantienen relaciones lejanas y, en todo caso, complejas.

La integración, palabra ambigua y polisémica, queda de todas formas en el interior de las lógicas funcionales de la sociedad receptora, y utiliza un repertorio que se basa en una mezcla de factores culturales y socioeconómicos, como queda de manifiesto en la propuesta de Portes y sus colegas (Portes, Fernández Kelly y Haller, 2009) en términos de asimilación segmentada; nos interrogamos sobre cómo el individuo es capaz de integrarse, pero no sobre cómo las instituciones son capaces de transformarse a sí mismas fagocitando nuevas biografías individuales y colectivas para la constitución de un espacio social y público. En el caso italiano parece curiosamente ejemplar la insistencia en la educación en la ciudadanía para los hijos de las migraciones, que se ve contrarrestada por la imposibilidad, o casi, del acceso a la ciudadanía legal. La legislación acerca del tema se refleja, por otro lado, sobre las percepciones que los jóvenes construyen en términos de sentido de pertenencia a un país; la elección en la legislación italiana del principio de sangre se configura por lo tanto como el dispositivo crucial que pauta una inclusión diferencial para un segmento mayoritario de la juventud de origen inmigrante.

Esta superposición entre sociedad, cultura y nación, que crea la figura de los italianos con una autorización de residencia, o, dicho de otra forma, de los extranjeros no inmigrantes, y hace difícil reconocer la existencia de la "identidades con guión", se refleja además en las maneras de pensar nuestro objeto de investigación en términos de nacionalismo metodológico. Por lo tanto, si la sociedad no coincide con la nación, el mismo tríptico a través del cual Marshall (1992) describió la dialéctica y la expansión de la ciudadanía (derechos civiles, políticos, sociales) nos devuelve un campo en el que están tachados/ excluidos una multitud de sujetos y de vidas enraizadas.

Volver a abrir la dialéctica sobre la ciudadanía significa interrogarse también sobre las representaciones y sobre las percepciones que los sujetos dan a este estatus Un trabajo reciente y original de Colombo, Domaneschi, Marchetti $(2009,2011)$ insiste en la importancia de registrar el nivel de las representaciones entre los hijos de la migración y sus pares italianos. Para los primeros la ciudadanía asume tanto un valor oportunista (una herramienta de acceso al derecho a la movilidad, a viajar, a quedarse), como representa una vía a la participación, una condición para el ejercicio de aquel derecho a la 
palabra que rompe la neutralidad del huésped, que se pretende a menudo en las sociedad receptora. Una importancia menor tiene, al contrario, la ciudadanía como identificación de tipo cultural, moral: como afirma uno de los chicos entrevistados en el marco de esta investigación, "Yo dentro puedo llegar a ser italiano, pero fuera no". Es decir, los trayectos de aculturación selectiva, de hibridación y de mestizaje, que crean los nuevos italianos se enfrentan con las miradas externas que están estructuradas todavía en las lógicas de las tres A: acento, ascendencia, apariencia (Ambrosini, 2005).

En este marco, una tensión moral en términos de ciudadanía como asimilación no parece organizar el nivel principal de las percepciones y representaciones entre los hijos de la migración, dado que es difícil transformar los límites de la Italianidad como comunidad imaginada, haciéndola más incluyente y volver a escribir el acceso a la ciudadanía más allá de los lazos de sangre. Además los hijos de la migración se apartan de forma creativa del discurso asimilacionista distinguiendo entre estilo de vida -acostumbrarse a ser jóvenes en Italia- y cultura que no apela tanto al origen étnico sino a la educación familiar. El resultado es sin duda una tensión participativa sin deseo de asimilación; en esta perspectiva, la ciudadanía está pensada más en términos de igualdad de oportunidades que de igualdad de identidades, y el pasaporte se convierte tanto en el instrumento de entrada en la cosmópolis como en la marca de la exclusión (Colombo, 2010).

Este plan de relaciones subjetivas, simbólicas y prácticas con la ciudadanía se compara, sin embargo, con un doble movimiento: por un lado por parte de los jóvenes nativos la idea de una condicionalidad (Colombo, 2011, White 2011) - ser italianos como algo que hay que merecerse para demostrar ser buenos ciudadanos - por el otro, el regreso en el pensamiento de Estado de un discurso asimilativo y de dispositivos relacionados (prueba de idioma, los acuerdos de integración, la ciudadanía como una recompensa) ${ }^{2}$.

Por lo tanto, podríamos repensar la condición juvenil en la línea de Mannheim (1928): las generaciones como grupos de edad que se definen y se forman relacionándose con una transformación histórica del mundo. Según la lectura de Cavalli (2008), consolidada en la literatura y en el sentido común, el debilitamiento de macro-eventos relevantes lleva a los jóvenes a una presentización de los horizontes temporales. En este terreno, sin embargo, los nativos italianos e hijos de la migración experimentan similitudes y diferencias. Educación de masas, precariedad laboral y prolongación de la separación de la familiaque son condiciones estructurales de la actual condición juvenil en Italia, se encarnan y declinan en el mismo grupo de edad de diferentes maneras por clase, $r a z a^{3}$

2. También es una tendencia difundida en muchos países europeos (tómense como ejemplo el caso de Holanda, en la que el conocimiento de la lengua se convierte en una condición para pedir el visado de entrada), que va de la mano con un énfasis en el poder del Estado para seleccionar otra vez la migración y separar desde arriba la buena y útil de la mala y poco rentable.

3. Para un desarrollo sobre el tema véanse la contribuciones de Curcio y Mellino (2010, 2012), así como la reciente edición de Mondi Migranti (Queirolo Palmas y Rahola, 2011). 
y nacionalidad; para los jóvenes de origen migrante la escolarización de masas, que se canaliza sobre todo en los institutos técnicos y profesionales, tiene unas tasas elevadas de expulsión ${ }^{4}$, la precariedad sufre de un surplus de explotación debida a la producción constante de irregularidades administrativas por parte del Estado, las relaciones sociales en el interior de las familias empujan hacia una rápida entrada en la edad adulta y a asumir una responsabilidad en el cuadro de la economía doméstica. Se trata de una triple discriminación que pesa sobre estos nuevos italianos: el ser jóvenes, migrantes y, por último, proletarios.

Por eso la tesis de la presentización no resulta convincente y, por el contrario, es oportuno recuperar la urdimbre compleja de la inclusión diferencial (simbólica, material, jurídica, administrativa, laboral, educativa), que se despliega en la trama de un modelo social ya no fordista, como el verdadero hecho de generación que construye social y políticamente a los hijos de la migración. Generación entendida como proceso que se adensa y precipita en torno a eventos y procesos significativos, algo que acontece y que, en su cristalización, cambia las formas de sentir, de ser, de relacionarse, de pensarse como individuos y como colectivos: puede ser el asesinato de Pym Fortuyin y Theo Van Gogh en Holanda y la siguiente ola de islamofobia, el asesinato por parte de la policía de un adolescente que se escapa y la revuelta de los suburbios franceses, el castillo kafkiano de autorizaciones de residencias y ciudadanías en muchos países, la segregación residencial que no presenta indicios de disminución, la alarma social sobre las pandillas como nuevo enemigo público en las ciudades, las primeras huellas dejadas en la comisaría, la crisis de un multiculturalismo pacificado en la ola de los disturbios de Londres, la imposibilidad que se le garantice el derecho a la movilidad... u otra cosa. Pero lo que importa es que estos son signos que marcan una generación, que la exponen públicamente y que, de forma básicamente similar en países con diferentes tradiciones migratorias (por el pasado colonial, antigüedad de residencia, modelos de gestión de la ciudadanía) articulan un discurso securitario y ponen en escena un enemigo público. Los hijos de la migración se enfrentan hoy en día con la reproposición de una lógica de la asimilación, pero también con una crisis estructural de las trayectorias de movilidad - aseguradas por la escuela y lafabrica, instrucción y trabajo, durante un largo periodo del siglo veinte- multiplicadas por procesos de clandestinización, encarcelamiento de masas (Oddone y Queirolo Palmas, 2011) e irregularización administrativa de sus derechos a existir, vivir, residir y viajar.

Sin embargo, como señala Lydia Lo Schiavo (2011: 2), "la ilusión colectiva de la temporalidad se enfrenta con la historicidad-subjetividad naciente de los jóvenes migrantes";

4. Según los datos de la publicación anual del MIUR (Ministerio de la Instrucción, Universidad e Investigación), en el año escolar 2008/2009 el 79,4\% de los estudiantes de la secundaria superior están matriculados en escuelas de formación profesional $(40,6 \%)$ y técnicos $(38,8 \%)$ 
será necesario, entonces, comenzar a esbozar algunos de estos campos en los cuales una subjetividad resistente y trasformadora comienza a tomar forma.

\section{PARA UNA LECTURA DE LA CONDICIÓN JUVENIL MIGRANTE EN TÉRMINOS DE AGENCIA Y RESISTENCIA}

La clave de nuestra interpretación radica en el concepto de agencia, como capacidad de protagonismo, de transformación creativa de las relaciones sociales, de resistencia infrapolítica a la inclusión diferencial. El espacio de la agencia se sitúa entre la condición del actor (el que interpreta un rol asignándole por un guión de relaciones sociales) y la condición del autor (el que transforma, establece el ritmo y el orden de los papeles del guión que hay que recitar). Es la dimensión de la improvisación que según Bourdieu actúa siempre en cada campo y rompe los vínculos entre el espacio de las posiciones y el espacio de las tomas de posiciones. La agencia, puede ser de tipo proyectivo, o sea, mirar hacia el futuro (Emirbayer, Mishe, 1998); en palabras de Brotherthon (2010: 33), "este tipo de agencia-resistencia comienza con pequeños gestos y actos polémicos dirigidos a las relaciones de poder existentes. Con el tiempo estos gestos, incluso los aislados, se convierten en una serie de acciones con efectos transformadores tanto para el sí como para el mundo social de los actores. Más en general, también podríamos describir esta iniciativa I resistencia como la oposición consciente o inconsciente de los individuos y grupos hacia limitaciones estructurales, tanto si se expresan en forma de valores y vínculos institucionales como si lo hacen en forma de micro-macro procesos de sometimiento cultural, psicológico, económico y social. [] En cualquier caso, las consecuencias de dicha resistencia son ambivalentes $y$ dependen del contexto local y del ambiente organizativo, que les confieren dirección y forma". Podemos localizar los hilos de la agencia en la actitud asertiva de las segundas generaciones en la reivindicación del rechazo de su condición de italianos con autorización de residencia, en el crecimiento de nuevas formas de asociacionismo no-étnico, en la denuncia no tanto de los déficit subjetivos de integración como de los dispositivos estatales que integran excluyendo, pero también en los usos tácticos, oportunistas e instrumentales de la diferencia, en la exhibición de los cuerpos y de las presencias, en las estéticas exhibidas, en las apropiaciones microfísicas de los espacios urbanos. Asimismo, es importante asumirla, de forma laica, en su modalidades más embarazosas: el trapicheo, el hurto, las prácticas predatorias en las calles representan, como ya subrayó Bourgois (2005) en una magistral etnografía, formas de agencias inmersas en la ambivalencia y mecanismos de reproducción social del destino que se tiene asignado. En una investigación reciente que hemos llevado a cabo en el recinto penitenciario más grande de Liguria, varios jóvenes de origen inmigrante reivindicaban su cursus criminal como única forma de resistencia contra la explotación laboral y la humillación social; Luis, por ejemplo, en lugar de ir a recoger tomates en los campos de Sicilia por unos pocos de euros prefería vivir de una migración estacional vinculada 
a la realización de robos en algunas grandes ciudades del norte, mientras que Ahmed, con un pasado como vendedor de rosas, que fue luego arrestado por posesión y venta de pequeñas cantidades de drogas, encontró en el trapicheo su rescate y su movilidad simbólica: "Cuando vendía flores, era yo que tenía que ir a preguntar a los italianos, cuando empecé a vender hachís, fueron los italianos que vinieron a verme, me necesitaban. Me sentí alguien, alguien que no pide, alguien más respetado". Este tipo de procedimientos de neutralización del estigma dan vida a los que Katz (1998), en una obra fundamental en la criminología crítica y cultural - The Seduction of Crime-, ha llamado las élites de la calle, grupos e individuos que a través de un trabajo político y simbólico acumulan respeto y reconocimiento en los círculos sociales que son importante para ellos.

Tal y como sugiere Colombo (2010), es el momento de superar la idea de déficit y división entre culturas para considerar importantes segmentos de esta generación hija de la migración como una minoría activa, una clase media marginal caracterizada por un elevado capital cultural familiar respecto del promedio de la población italiana (capital sin embargo desvalorizado en términos de colocación laboral), por el mantenimiento de relaciones fuertes - pero también de rescritura cultural - con la red y los orígenes familiares, por los contactos frecuentes y trasversales con los nativos; jóvenes por un lado afectados por múltiples procesos de discriminación marcados por el renacimiento de una línea del color ${ }^{5}$ (Du Bois, 2007) y, por otro lado, capaces de moverse en las porosidades del espacio social, contando con la ambivalencia como recurso y como respuesta creativa a los vínculos materiales y simbólicos, para colocarse de forma ventajosa en el interior del hacerse global del mundo.

Esta dimensión de agencia / resistencia, transversal a las generación de la que estamos hablando, sin embargo, está fuertemente marcada por las líneas de clase y se articula en la tensión entre clase media marginalizada y proletariado juvenil migrante: por un lado está quién ha intentado convertir el capital cultural familiar, privado de valor en el mercado laboral italiano, en nuevas inversiones educativas, por el otro lado quien, expulsado por los circuitos de la formación, resiste de manera creativa o sucumbe a lo que, para el caso norteamericano, Loic Wacquant (2001) ha llamado la simbiosis mortal entre el gueto y la cárcel. La línea de color les afecta a todas / os y para ambos grupos vemos operar formas y prácticas de contestación; es un excelente ejemplo de esta polaridad, la distancia, en términos de lenguajes, deseos, expectativas, estéticas, consumos, formas de voice entre el mundo asociativo de las redes G2 (en su mayoría estudiantes universitarios con una performance de la lengua italiana no contaminada por la herencia migratoria) y el mundo de las pandillas y de las organizaciones de la calle (cuyos miembros despliegan

5. Por línea de color, siguiendo a Du Bois, entendemos todas aquellas formas de estratificación del espacio social en donde la "raza" es construida simbólica y materialmente como una criba y un dispositivo de acceso a los recursos (empleo, educación, sanidad y prestigio). 
su propia socialidad en un contexto de relaciones sociales fuertemente marcadas por las vivencias migratorias, prefieren el uso de la lengua de origen como medio de distinción e invisibilización, y construyen narraciones individuales y colectivas desvinculadas de cualquier discurso sobre la italianidad como campo de reivindicación).

Pensar en las diferentes formas de agencia de los hijos de las migraciones significa entonces pensar las diferencias no como cosas, sino como estrategias, un repertorio de prácticas que se utilizan de forma contingente, y que articulan ironía, mimetismo, ostentación, énfasis e itinerancia. Esta perspectiva, en términos de multiculturalismo cotidiano, que está lejos de cualquier idea de cultura como contenedor especifico, esencializado y naturalizado que hay que legitimar y organizar a través de políticas culturales de reconocimiento especificas (Colombo y Semillas 2007), es crucial para empezar a cubrir uno de los vacíos principales de la investigación sociológica sobre el tema: sabemos mucho de la participación escolar de los jóvenes de origen extranjero, aún sabemos muy poco acerca de sus diversas culturas de generación y de las prácticas de acceso y voz en el espacio público.

Sayad (2002) siempre nos recuerda que el racismo existe y se percibe sólo cuando se sale de la cálida acogida del círculo étnico-nacional-familiar, cuando se dan los primeros pasos en las sociedades de llegada. La generación de la que hablamos ha crecido dentro esta Italia, no puede ser expulsada, abortada o re-colocada y etnizada en la medida en que se niega aceptar un destino asignado. En el marco de una investigación europea Benasso, Cortellesi y Villa (2010) han observado en el caso italiano como los jóvenes de origen extranjero entrevistados por un lado consideraban ser percibidos en función de sus propios orígenes (nacionales, étnicos, culturales, religiosos) y, por otro, exponían formas de auto-identificación relacionadas con la edad (ser joven o adolescente), a los gustos musicales, a las estéticas, a las prácticas deportivas y a los estilos sexuales. En esta perspectiva, la línea de color, dispositivo de control de la movilidad material y simbólica de una generación, es la reacción póstuma a la invasión: la de quien pone en cuestión con sus propias prácticas la herencia de la condición migrante y se considera, viceversa, hijo de su propia vida en lugar de "hijo de la migración".

En el campo educativo ( $\sin$ duda uno de los más frecuentados por la literatura), a pesar de los efectos estructurales de un confinamiento de clase dentro de la educación técnica y profesional, diversos estudios han puesto de relieve otra narrativa posible. La experiencia de la escuela es crucial para determinar los horizontes de la vida, porque en ella se condensan y se reflejan múltiples prácticas de inclusión y exclusión. La escuela a través de la acción desde abajo de miles de actores (profesores, directores, familias, asociaciones, mediadores culturales) ha creado una configuración latente: la inserción en la escuela de todos, la irrelevancia de la condición jurídica del menor, la centralidad de la persona, una orientación positiva (aunque a veces ambigua o ingenua) hacia la 
intercultura, la centralidad de la relación con las familias. La atención a los accesos, a menudo, no se ha acompañado de una atención a los éxitos, a las intervenciones para contrarrestar la dispersión y el apoyo a una orientación en condiciones de igualdad en los inicios de la escolarización. (Giovannini y Queirolo Palmas, 2002; Chaloff y Queirolo Palmas, 2006). La investigaciones invitan además a superar el pensamiento binario, evitando pensar en los estudiantes de origen extranjero como un ellos para contrastar metodológicamente y teóricamente con un nosotros, asumiendo la pluralidad de factores (de clase, de capital cultural, de condiciones familiares, de climas escolares, de estilos de enseñanza) que intervienen en las trayectorias educativas (Giovannini y Queirolo Palmas, 2002). Si descomponemos la condición de los alumnos de origen migrante a la luz de la heterogeneidad, incluso las proclamas recientes acerca de los riesgos de la concentración de las presencias - desde la propuestas de aulas-puentes a la identificación de techo máximos definidos además por criterios ambiguos- se inscriben en la retórica de la alarma y de la producción de imaginarios de exclusión.

Se debe otra vez volver a retomar la migración como un espejo, como un proceso que trabaja y gira en el conjuntote la sociedad, transformándola y restituyendo una imagen que va más allá del objeto de estudio específico; observar las migraciones en las escuelas por lo tanto, nos ayuda a entender el funcionamiento general de estos espacios y estas instituciones, más que revelarnos las características peculiares y folklóricas del objeto estudiado.

A pesar de los datos agregados sobre el éxito, los retrasos y los abandonos señalan una disparidad estructural entre extranjeros y no extranjeros, como nos sugiere Besozzi (2011: 53), "los estudios recientes que comparan las primeras y segundas generaciones (Casacchia, Natale, Paterno y Terzera, 2008; Gilardoni, 2008; Thieghi y Ognissanti, 2009; Luciano, Ricucci y De Martini, 2009) ponen claramente de relieve como las segundas generaciones están, de hecho, más cerca de los coetáneos italianos, tanto en comparación con el rendimiento académico como en la intención de continuar sus estudios, mientras que los estudiantes recién llegados y insertados en la edad de la adolescencia presentan más dificultades, con repeticiones y retrasos, y más a menudo deciden abandonar la escuela o bien optar por una opción de elección laboral temprana después de la escuela media." Otros estudios también han documentado un efecto del género en términos de éxito entre los hijos de la inmigración (Ravecca, 2009).

Esta heterogeneidad en el campo educativo dibuja, siguiendo los resultados de un estudio reciente sobre más de mil alumnos extranjeros de escuelas lombardas y basado en un cluster analysis (Besozzi, Colombo, Santagati 2009), una pluralidad de tipos y situaciones: un grupo con bajo nivel de inversión / motivación y fuerte fracaso (25,3\% de la muestra), un segundo grupo con un nivel de inversión realista de carácter instrumental (33,8\% de la muestra), que encuentra en un buen éxito escolar en la educación técnica y 
profesional la manera de acceder al mercado laboral y, por último, un grupo explorativo (40,9\% de la muestra) que en su mayoría privilegia la opción de la continuación en el ámbito universitario y desafía el reto de la integración subalterna a la cual está anclada la vida de las familias respectivas. Estos resultados, sin negar las dificultades y los procesos de discriminación, devuelven un cuadro difícilmente subsumible bajo el signo de la pasividad y del déficit cultural como condición ontológica.

En cualquier caso, para todos y todas, debemos preguntarnos no porqué ciertos grupos de jóvenes tienen dificultades en la utilización de las oportunidades educativas, sino sobre los factores que hacen que las instituciones educativas sean poco eficaces en el apoyar el éxito y el acceso escolar de los hijos de la migración, y más en general de los hijos de las clases populares.

Comprender la experiencia escolar significa también investigar la experiencia extracurricular, las relaciones entre las culturas juveniles empezando por la consolidación de los flujos migratorios y de la circulación de imaginarios vehiculados por las industrias culturales globales. En esta perspectiva, la investigación se ha enfrentado al tema de las participaciones y de las percepciones de ciudadanía y pertenencia (Colombo et al., 2009), el papel del capital social en la determinación de los resultados educativos (Ravecca, 2009), la importancia de la religiosidad (Frisina , 2007), las experiencias de discriminación entre los italianos con guión (Andall, 2003), las apropiaciones lúdico-deportivas del espacio público (Zoletto, 2010) y la racialización de los gimnasios (Marengo 2011), y los consumos como fuente de identidad ( Leonini, 2008).

Las investigaciones sobre pandillas (Queirolo Palmas, 2009, 2010) han permitido entender, en un contexto de gran vulnerabilidad social y existencial, el desarrollo de los mecanismos de protagonismo y de creatividad, que de inmediato ponen en crisis cualquier visión patologizante: la inversión de la doble ausencia en doble presencia, la apropiación y re-significación de los espacios urbanos (home making), la transición de la condición de no-persona a la de hiper-persona a través del juego de los excesos y de una estética espectacular, la creación de códigos y lenguajes secretos con el fin de inmunizarse a las miradas hostiles, la transformación del estigma en emblema. Estas formas de creatividad social pasa a través del re-naming (Brotherton y Barrios 2004), los procesos narrativos capaces de re-significar el mundo social empezando por las necesidades del grupo y luego construir un nosotros que tenga respeto. Los Latin Kings, Ñetas, Maras, Vatos Locos y un sinnúmero de otras denominaciones exóticas, dibujan así formas de pertenencia post-nacional, inventan naciones y proyectan las vida de los seguidores bajo la mitología de una Raza Latina, tanto indistinta como permeable a todos, declinan un lenguaje impregnado de criterios familiares - la familia, los hermanitos - y rituales religiosos - el ayuno, las oraciones, los rosarios, las canciones, etc ... - para fortalecer el espíritu de una hermandad juvenil mestiza. Como nos dice Tupac, veinte años, peón en 
la construcción y ex líder de la Nación de los Latin King en Génova. “Todo el mundo es aceptado aquí, no importa el color de tu piel si eres gordo o flaco, si tienes ropa a la moda o no, si eres rico o si eres pobre, si eres hombre o mujer, si eres italiano o extranjero. Para algunos somos sólo un pedazo de papel, una autorización de residencia, una tarjeta para enseñar. Pero nosotros somos gente como vosotros. La Nación de los Latin King es como una religión que te hace creer en ti mismo. Enseñamos a los hermanitos (los miembros) a resistir, a enfrentarse a sus verdaderos problemas. No somos una banda somos un movimiento revolucionario".

Deberíamos entonces seguir mapeando los signos de estas incursiones en los diversos campos dentro los cuales toma forma la subjetividad y la historicidad de la generación de la cual escribimos: la expresividad en la música y el arte, en las iglesias evangélicas y en las mezquitas, en las parroquias de los boys scouts o en los centros sociales ocupados, en el compromiso sobre la inversión en la educación, en los gimnasios populares, en la regeneración desde abajo de los espacios públicos urbanos, en los inventos de identificaciones espectaculares realizadas por las pandillas juveniles, en las rampas de los skaters o en los muros de grafitis emerge gradualmente una red de relaciones y de sociabilidad que circunvala la línea de color, atravesando formas de resistencia que juegan en la ambivalencia.

Frente a un estigma que se organiza de forma móvil construyendo socialmente la raza y el color - inventando la raza blanca, la raza negra y una multiplicidad de razas y cristalizaciones intermedias del color- los jóvenes hijos de la migración articulan prácticas de resistencia distinta: de la transformación del estigma en emblema (el orgullo mestizo, la blackness, la raza latina), al mimetismo, a los gestos oblicuos y a la reivindicación de un derecho a la indiferencia. En una investigación reciente llevada a cabo en Barcelona (Giliberti, 2011), jóvenes dominicanos migrantes acostumbrados a pensarse a sí mismos como blancos (en relación a los haitianos como significante negro) descubren que ellos son tratados como negros en la sociedad de llegada; el color, la raza, aparece en las palabras de uno de los informantes como una clara etiqueta móvil aplicada a la explotación "los negros trabajan como negros porque los negros son siempre los que trabajan más, para vivir como blancos ...". Frente a la racialización estos jóvenes comienzan por un lado a acumular capital simbólico reivindicando su orgullo de ser los negros de Barcelona, por el otro, a oponerse a cualquier etiqueta actuando sobre el terreno del derecho a la indiferencia. Otros, al contrario, internalizan el desvalor de ser considerado negro e intentan encontrar nuevos objetivos y nuevos grupos sociales sobre los cuales dirigir el estigma.

Asumir la agencia de los jóvenes y de la segunda generación significa por lo tanto sobrepasar el debate mismo sobre la segunda generación, término que reduce el espacio de la sociabilidad a un origen y una descendencia, obliterando las capacidades creativas 
de los sujetos para desafiar el pensamiento de Estado, la posteridad inoportuna, la línea del color. Es sobre estos terrenos que los jóvenes dejan de ser inmigrantes, liberándose de los residuos de la doxa científica y popular que quisiera encerrarlos en una "lectura tranquilizante" en términos de cohesión social, déficit y pasividad, comunitarismo étnico y suspensión entre universos culturales. 


\section{REFERENCIAS BIBLIOGRÁFICAS}

Ambrosini, Maurizio (2005) Sociologia delle migrazioni. Bologna: Il Mulino.

Ambrosini Maurizio (2009) "Nuovi concittadini? I giovani di origine immigrata vettore di cambiamento della società italiana”. Altre Modernità, 2, pp. 20-28.

Andall Jaqueline (2003) "Italiani o stranieri? La seconda generazione in Italia". En Sciortino G. y A. Colombo, Un'immigrazione normale. Bologna: Il Mulino.

Barbagli Marzio (2002) Immigrazione e reati in Italia. Bologna: Il Mulino.

Barban, N.; Conti, C.; Gabrielli, D. y Guarneri A. (2011) "I nuovi italiani di tanti colori", $\mathrm{X}$ conferenza nazionale di statistica, en linea: http://www.slideshare.net/slideistat/ barban-7694267

Baumann, Gerard (2003) L'enigma multiculturale. Stati, etnie, religioni. Bologna: Il Mulino.

Benasso S.; Cortellesi G. y Villa A. (2010) "Crossing Sights: migrant youth in two Italian cities". Italian Journal of Sociology of Education, 4 (1).

Besozzi, Elena (2011) "Il successo scolastico dei minori stranieri fra prima e seconda generazione”. Libertà Civili, gennaio-febbraio.

Besozzi, E.; Colombo, M. Y Santagati, M. T. (2009) Adolescenti stranieri, nuovi cittadini. Le traiettorie di vita di una generazione ponte. Milano: Franco Angeli.

Bianchi, Giorgia (2011) "Italiani nuovi o nuova Italia, Citizenship and Attitudes towards the Second Generation in Contemporary Italy”. Journal of Modern Italian Studies, 16 (3), pp. 321-333.

Bourdieu Pierre (2003) Per una teoria della pratica. Con tre studi di etnologia cabila. Milano: Cortina.

Bourgois, Philippe (2005) Cercando rispetto. Drug economy e cultura della strada. Bologna: Derive e approdi.

Brotherton, David (2010) "Oltre la riproduzione sociale. Reintrodurre la resistenza nella teoria sulle bande". En Queirolo Palmas (ed) Atlantico latino: gang giovanili e culture transnazionali. Roma: Carocci.

Brotherton, David y Barrios, Luis (2004) The Almighthy Latin King and Queen Nation. Street politics and the Ttransformation of a New York City Gang. New York: Columbia University Press. 
Caneva, Elena (2011) Mix generation. Gli adolescenti di origine straniera fra globale e locale. Milano: Franco Angeli.

Caritas/Migrantes (2009) Immigrazione. Dossier Statistico 2009. XIX Rapporto. Roma: Idos Edizioni.

Casacchia O.; Natale L.; Paterno A. y Terzeria L. ( a cura de) (2008) Studiare insieme, crescere insieme?. Milano: Franco Angeli.

Cavalli, Alessandro (2008) "Caratteri, risultati e prospettive delle ricerche sui giovani". En Rauty R. (a cura de) La ricerca Giovane. Percorsi di analisi della condizione giovanile. Lecce: Kurumuny.

Chaloff, Jonathan. y Queirolo Palmas, Luca ( a cura de) (2006) Scuole e migrazioni in Europa. Dibattiti e prospettive. Roma: Carocci.

Colombo, Enzo (a cura de) (2010) Figli di migranti in Italia. Identificazioni, relazioni, pratiche. Torino: Utet.

Colombo, E.; Domaneschi, L. y Marchetti C. (2009) Una nuova generazione di italiani. L'idea di cittadinanza fra i giovani figli di immigrati. Milano: Franco Angeli.

Colombo, E., Domaneschi, L. y Marchetti, C. (2011) “Citizenship and Multiple Belonging. Representation of Inclusion, Identification and Participation among Children of Immigrants in Italy" Journal of Modern Italian Studies 16 (3), pp. 334-347.

Colombo, Enzo y Semi, Giovanni (2007) Multiculturalismo quotidiano. Le pratiche della differenza. Milano: Franco Angeli.

Curcio, Anna y Mellino, Miguel (2010) "Race at work - the rise and challenge of Italian racism". Darkmatter, 6.

Curcio, Anna y Mellino, Miguel (2012) (a cura de) La razza al lavoro. Roma: Il Manifesto Libri.

Delgado, Manuel (2007) Sociedades Movedizas. Pasos hacia una antropologia de la calle. Barcelona: Anagrama.

Delgado, Manuel (2010) "Gli studi sulle migrazioni in Spagna. Un bilancio e alcune riflessioni”. En Salvatore Palidda (a cura de) Il discorso ambiguo sulle migrazioni, Messina: Mesogea.

Diaco, A.; Mota Santos, P.; Morago, H. y Teodorani, E. (2009) In between. 9 Takes from the european scene. Genova: Carta - Laboratorio di Sociologia visuale, Genova - Roma. 
Djouder, Ahmed (2007) Disintegrati. Storia corale di una generazione di immigrati. Milano: Il Saggiatore.

Du Bois, Williams E. B. (2007) Le anime del popolo nero. Firenze: Le lettere.

Emirbayer, Mustafa y Mishe, Ann (1998) "What is agency". The American Journal of sociology, 103 (4), pp. 962-1023.

Frisina, Annalisa ( 2007) Giovani musulmani d'Italia. Roma: Carocci.

Gans, Herbert (1992) "Second-generation decline: Scenarios for the economic and ethnic futures of the post-1965 American Immigrant". Ethnic and Racial Studies, 15 (2).

Gilardoni, Guia (2008) Somiglianze e differenze. Lintegrazione delle nuove generazioni nella società multiétnica. Milano: Franco Angeli.

Giliberti, Luca (2011) "Negri di Barcellona. Giovani dominicani tra stigma e resistenza". Mondi Migranti, 3.

Giovannini Grazziella y Queirolo Palmas Luca (a cura de) (2002) Una scuola in comune. Esperienze scolastiche in contesti multietnici italiani. Torino: Edizioni della Fondazione Agnelli.

Glissant, Edouard (2007) Poetica della relazione. Macerata: Quodlibet.

Golini, Antonio (2011) "I minori stranieri. Una galassia difficile da definire e quantificare". Libertà Civili, gennaio-febbraio.

Katz, Jack (1988) Seductions of crime. Moral and Sensual Attractions in Doing Evil. New York: Basic Books.

Leonini, Luisa (2008) “Consumi e identità”. Mondi Migranti, 3.

Lo Schiavo, Lidia (2011) "Sfera pubblica, giovani migranti, intersezionalità: alcuni elementi di analisi”. Quaderni di intercultura, 3.

Luciano, A.; Demartini, M. y Ricucci, R. (2009) "Istruzione dopo la scuola dell’obbligo? Quali percorsi per gli alunni stranieri?”. En Giovanna Zincone (a cura de) Immigrazione: segnali di integrazione. Sanità, scuola e casa. Bologna: Il Mulino.

Mannheim, Karl (2008) [1928] Le Generazioni, Il Mulino, Bologna

Marengo, Gilberto (2011) "Dal daltonismo sportivo all'inclusione strabica. Migranti e capitale guerriero in una palestra di kickboxing". Mondi Migranti, 3. 
Marshall, Thomas H. (1992) Citizenship and Social Class. London: Pluto Press.

Martiniello, Marco (2000) Le società multietniche. Bologna: Il Mulino.

Mezzadra, Sandro (a cura de) (2004) I confini della libertà. Per unanalisi politica delle migrazioni contemporanee. Roma: DeriveApprodi.

Ministero degli Interni (2010) Dati sulla cittadinanza, annate varie. En la web http://www. interno.it/mininterno/export/sites/default/it/temi/cittadinanza/sottotema008.html

Naletto, Grazia (2009) Rapporto sul razzismo in Italia. Roma: Manifestolibri.

Oddone, Cristina y Queirolo Palmas, Luca (2011) "Dalle Gang al carcere. Vissuti della detenzione". Studi sulla questione criminale, 1.

Portes, A.; Fernandez Kelly, P. y Haller, W. (2009) "The Adaptation of the Immigrant Second Generation in America: a Theoretical Overview and Recent Evidence". Journal of Ethnic and Migration Studies, 35 (7).

Queirolo Palmas, Luca (a cura de) (2009) Dentro le gang. Giovani, migranti e nuovi spazi pubblici. Verona: Ombre Corte.

Queirolo Palmas, Luca (2006) Prove di seconde generazioni. Giovani di origine immigrata fra scuole e spazi urbani. Milano: Francoangeli.

Queirolo Palmas, Luca (a cura de) (2010) Atlantico Latino: gang giovanili e culture transnazionali. Roma: Carocci.

Queirolo Palmas, Luca y Rahola, Federico (2011) "Nominare la razza”. Mondi Migranti, 3.

Ravecca, Andrea (2009) Studiare nonostante. Capitale sociale e successo scolastico degli studenti di orgine immigrata nelle scuole superiori. Milano: Franco Angeli.

Rumbaut, Ruben (1997) "Assimilation and its Discontents: between Rhetoric and Reality". International Migration, 4.

Saitta, Pietro (2011) "Between Kafka and Carnevale: an introduction to the Immigrant Condition in Italy". Journal of Modern Italian Studies, 3.

Sayad, Abdelmalek (2002) La doppia assenza. Dalle illusioni dellemigrato alle sofferenze dell'immigrato. Milano: Raffaello Cortina.

Sayad, Abdelmalek (2008) L'immigrazione o i paradossi dell'alterità. Verona: Ombre Corte. 
Thieghi, Laura y Ognisanti, Mirca (a cura de) (2009) Seconde generazioni e riuscita scolastica. Milano: Franco Angeli.

Wacquant, Loïc (2001) "Deadly Symbiosis: When Ghetto and Prison Meet and Mesh". Punishment \& Society 3 (1).

Wrench, J.; Rea, A. y Ouali, N. (eds) (1999) Migrants Ethnic Minorities and the Labour Market, Integration and Exclusion in Europe. London: Macmillian press.

Zoletto, Davide (2010) Il gioco duro dellintegrazione. L'intercultura sui campi da gioco. Firenze: Raffaello Cortina. 